\title{
Padrão de atividade física em crianças e jovens: Um breve resumo do estado do conhecimento
}

\author{
Physical activity patterns in children and youth: a brief summary \\ of current knowledge
}

\author{
Ramon de Brito Costa Pinheiro Lima ${ }^{1}$ \\ Rogério César Fermino ${ }^{2,3}$ \\ André Seabra ${ }^{1}$ \\ Rui Garganta ${ }^{1}$ \\ José António Ribeiro Maia ${ }^{1}$
}

\footnotetext{
1 Universidade do Porto. Faculdade de Desporto. Laboratório de Cineantropometria e Estatística Aplicada. Porto. Portugal.

2 Universidade Federal do Paraná. Programa de Pós-Graduação em Educação Física. Curitiba, PR. Brasil.
}

3 Pontifícia Universidade Católica do Paraná. Grupo de Pesquisa em Atividade Física e Qualidade de Vida. Curitiba, PR. Brasil.

Recebido em 05/11/08 Revisado em 27/02/09 Aprovado em 24/06/09

\begin{abstract}
Resumo - O objetivo principal desta revisão foi abordar aspectos conceituais, operacionais e apresentar os principais resultados relativos ao padrão de atividade física (PAF) em crianças e jovens. Foi realizada uma pesquisa em bases de dados nacionais e internacionais com intuito de selecionar estudos originais recentes que apresentassem informações acerca do PAF. A maioria das pesquisas apresentam maneiras distintas de conceituar e operacionalizar o PAF, utilizando técnicas estatísticas univariadas e multivariadas. Grande parte dos estudos identificou que a atividade física na infância se caracteriza por períodos extremamente curtos de alta intensidade, com predomínio de atividades de moderada à baixa intensidade. Durante os finais de semana, esses períodos são maiores e os meninos apresentam uma maior frequência de episódios de níveis distintos de intensidade. A expressão "padrão de atividade física" é comumente utilizada, porém não é encontrada uma interpretação e conceitualização unânime. A adoção de análise multimodal seria uma mais valia, uma vez que poderá auxiliar o entendimento das nuances da atividade física, considerando o seu tipo, frequência, duração e intensidade.
\end{abstract}

Palavras-chave: Atividade física; Padrões; Crianças; Adolescentes.

Abstract - The main purpose of this review was to accost conceptual and operational issues associated with physical activity patterns (PAF) in children and adolescents. A systematic research was done in national and international data bases in order to select recent studies with information concerning PAF. There are different ways to describe and quantify PAF using univariate and multivariate statistical techniques. Most of studies identified that physical activity is characterized by extremely short periods of high intensity, with a predominance of moderate to low intensity activity. On weekends these periods are longer and boys have higher frequencies of episodes in different levels of intensity. "Physical activity pattern" is a usual expression, but there is no unanimous concept and interpretation. Using a multimode approach would certainly improve our understanding of PAF, considering its type, frequency, duration, and intensity. Key words: Physical activity; Patterns; Children; Adolescents. 


\section{INTRODUÇÃO}

O crescimento desregrado da inatividade física na sociedade moderna tem proporcionado um aumento de diversas morbilidades e um incremento da mortalidade associada a doenças cardiovasculares ${ }^{1}$. A descrição exaustiva e as consequências deste fenômeno têm sido muito estudadas, em especial, na área da epidemiologia da atividade física $(\mathrm{AF})^{2,3}$.

É difícil contestar o benefício da prática moderada a vigorosa de AF para a promoção da saúde, qualidade de vida e longevidade ${ }^{1,2}$. Diversos estudos mostraram que as crianças apresentam baixos níveis de AF em consequência do aprimoramento dos meios de transporte e da forte atração por atividades sedentárias, como os jogos de computador e televisão $0^{3,4}$. Um comportamento fisicamente ativo na infância e adolescência apresenta forte probabilidade de repercutir-se na idade adulta ${ }^{5,6}$. Daqui que não sejam de estranhar as mais diversas recomendações de organizações oficiais que visam determinar os níveis de AF diária benéficos à saúde. As recomendações mais atuais encontradas foram sugeridas por Strong et al. ${ }^{1}$ : crianças e adolescentes devem realizar 60 minutos diários de AF com intensidade moderada-vigorosa, para que esta possa proporcionar benefícios à saúde. Os autores abordam a AF como um forte preditor de saúde no presente e no futuro, recomendando, desta forma, uma frequência diária de intensidade moderada a vigorosa de 60 minutos.

Se por nível de AF se entende a expressão, em termos absolutos ou relativos, de realização de atividades voluntárias medidas em tempo, METs, Kcal ou em outras unidades, não é suficientemente preciso a definição de padrão de $\mathrm{AF}$ (PAF) (do inglês physical activity pattern), não obstante existir modos distintos de operacionalizar tal conceito. Em língua inglesa ${ }^{7}$, padrão (pattern) é genericamente descrito como "a model or design used as a guide", ou "the change in sense from the idea of a model giving an example to be copied". A consulta ao dicionário da língua portuguesa ${ }^{8}$ apresenta, entre outras, as seguintes possibilidades:

a) modelo de referência para avaliação, uma norma ou um tipo ideal;

b) coisa ou palavra que serve de referência, regra ou princípio usado como base de julgamento;

c) coisa estabelecida por autoridades como regra para medida de quantidade, peso, comprimento, valor, ou qualquer coisa mensurável.
A palavra padrão tem origem latina, patronus ou o protetor dos pebleus. Vejamos a possibilidade de extensão semântica destas referências no domínio da AF. Será que apresentar um PAF adequado é protetor ou reflete um patronus saudável, capaz de permitir a realização de atividades diárias com vigor? A configuração das AF's é relativamente sistemática e intencional com dispêndios acima dos três METs? A sua forma, ainda que variável, será representada por um modelo no qual há duração suficiente de AF por mais de 10 minutos de intensidade moderada a vigorosa? Haverá uma representação única, ou ideal, de PAF, ou existe grande variação em termos intra e interindividuais? Haverá uma forma que permita efetuar algum julgamento relativo ao que se considera aceitável para cumprir com o compromisso de 60 minutos de AF diários? Estas são perguntas por esclarecer na literatura.

Apesar de existirem evidências sobre o nível de AF em termos populacionais e dos respectivos determinantes ${ }^{9}$, não está disponível informação sintética sobre a problemática do PAF em termos do seu conceito, operacionalização, métodos de análise e relevância dos resultados. Assim, os objetivos desta breve revisão foram (1) apresentar o conceito de PAF; (2) rever as principais formas da sua operacionalização; (3) identificar as técnicas de análise estatística mais usadas na sua descrição, e (4) apresentar os principais resultados relativos ao PAF de crianças e jovens.

\section{PROCEDIMENTOS METODOLÓGICOS}

Para referenciar a informação mais relevante, foi realizada uma pesquisa sistemática nas bases de dados Scielo e Lilacs com os descritores "padrão de AF", "crianças", "jovens" e "adolescentes" e na Pubmed com os correspondentes termos em inglês. Foram revisados estudos nos diversos domínios da AF e deveriam preencher os seguintes critérios de inclusão: (1) ter obrigatoriamente uma análise dos PAF, (2) serem artigos originais, (3) publicados a partir do ano 2000, (4) considerar exclusivamente crianças e jovens e, (5) avaliar a AF com questionários, diários, pedômetros, acelerômetros e monitores de frequência cardíaca $(\mathrm{FC})$.

\section{RESULTADOS}

\section{Conceito de padrão de atividade física}

É notadamente claro o fato da expressão PAF ser largamente utilizada, embora uma definição clara e 
concisa do que se entende por essa expressão, não só no plano conceitual mas também no plano operativo, não estar claramente apresentada. De acordo com alguns autores ${ }^{10-12}$, o PAF é caracterizado por um padrão intermitente de atividades de baixa intensidade. Para Lopes e Maia ${ }^{13}$ o PAF está intimamente ligado a aspectos da frequência, duração, intensidade e tipo de AF. Buchowski et al. ${ }^{14}$ referem que o PAF pode ser conhecido a partir do tempo despendido na realização de diferentes atividades com intensidades variadas e/ou não relacionadas diretamente com a sua intensidade. De acordo com Lopes et $a^{15}$, PAF é AF habitual, sendo esta definida como um comportamento complexo que tem por base hábitos e práticas individuais que variam consideravelmente a cada dia, estação climática e ano. Para Fox e Riddoch ${ }^{16} \mathrm{o}$ PAF é a AF habitual que os indivíduos apresentam no seu dia-a-dia, e está inteiramente relacionada com a intensidade e o tipo de atividade realizada. Magalhães et al. ${ }^{6}$ salientam a aleatoriedade e variabilidade do PAF em crianças, devido à sua espontaneidade, $\mathrm{O}$ que demonstra a natureza transitória da AF, oscilando na sua intensidade e com intervalos de duração muito irregulares. De acordo com os estudos acima mencionados, nota-se que não existe uma noção precisa do que se entende por PAF. Contudo, parece ser consensual a necessidade de se considerar aspectos como duração, frequência, intensidade, tipo, local e estação climática para melhor compreender o seu conteúdo, expressão e dinâmica.

\section{Operacionalização e formas de análise do padrão de atividade física}

É unanimemente aceito a noção de que a AF é um comportamento multifacetado e multidimensional que inclui aspectos como duração (minutos, horas, etc.), frequência (vezes por semana), intensidade (energia despendida por unidade de tempo), tipo ou modo (AF no lazer, transporte, etc) e relevância psicológica e social. A sua expressão quantitativa e qualitativa está condicionada aos instrumentos utilizados ${ }^{12,16}$.

Na maioria dos estudos, os pesquisadores utilizaram o acelerômetro para melhor medir a AF e assim expressar o seu padrão. A classificação da intensidade da AF tem sido categorizada como leve, moderada, vigorosa e muito vigorosa ${ }^{17}$. Porém não deixa de ser relevante mencionar o fato de outros autores $^{18,19}$ classificarem a AF de formas distintas, como por exemplo, em atividade leve, ligeira, sedentária, moderada e vigorosa, bem como o fato de utilizarem diferentes valores de corte.

As tabelas 1 à 3 apresentam as informações mais relevantes acerca dos estudos realizados em diferentes países. Parece-nos importante referir alguns trabalhos pela sua relevância e modo como conceberam a categorização dos PAF para salientar a sua diversidade conceitual e operativa.

Magalhães et al. ${ }^{6}$ procuraram identificar os níveis de AF no lazer e o PAF de crianças do quarto ano de escolaridade. A avaliação de AF no lazer foi realizada por um questionário que permite classificar a AF em leve, moderada e vigorosa, e pelo acelerômetro monitorando cinco dias escolares da semana, sendo considerado o tempo gasto em qualquer atividade para, posteriormente, calcular o dispêndio energético. Ndiaye e Benéficé ${ }^{20}$ utilizaram um acelerômetro e classificaram a AF em quatro níveis (repouso, leve, moderada e vigorosa), procurarando analisar minutos de atividade. Bathrellou et al. ${ }^{21}$ utilizaram um questionário para estudar os níveis de AF de crianças de escolas públicas de regiões urbanas e rurais de Chipre. Este registro ocorreu durante uma semana escolar e no final de semana e, em seguida, as atividades foram classificadas de acordo com a frequência e duração das AF do quotidiano e em comportamentos sedentários. Para avaliar o tempo individual despendido, foi utilizada uma escala com quatro níveis de resposta, variando de zero a mais de seis vezes por semana. Atividades esportivas (ciclismo, basquetebol, voleibol, corrida, futebol, etc) foram classificados como moderadas ou vigorosas. Jago et al. ${ }^{19}$ estudaram o PAF de acordo com o gênero, dia e horário com auxílio de um acelerômetro. Para a análise, foram considerados três momentos: antes ou durante o período escolar, tarde e noite. Com o intuito de obter informação detalhada, os sujeitos foram instruídos a preencher um diário contendo todas as atividades executadas. No estudo realizado por Pan e Frey ${ }^{17}$, com o objetivo de determinar o PAF de crianças com desordens do espectro autista, foi utilizado o acelerômetro durante sete dias consecutivos. Os aparelhos foram programados para coletar dados com intervalos de um minuto. As "activity counts" foram analisadas para determinar o total de movimentos em counts, counts por minuto e tempo utilizado em atividades ligeiras, moderadas, vigorosas e muito vigorosas. Os dados foram divididos em dois períodos ao longo dos dias escolares: durante (9-14 hrs) e após a escola (15-18 hrs). Os autores utilizaram, também, um questionário, contendo 21 itens para avaliar a AF diária.

\section{Análise estatística}

Convém salientar que a análise estatística da noção operacional de PAF tem sido efetuada de modo muito díspar, requerendo técnicas de natureza 
univariada, bivariada e multivariada que vão de simples tabelas de frequência até procedimentos mais sofisticados de análise da função discriminante e análise de variância multivariada. Ora nem sempre é clara a justificação de tanta variedade na análise dos dados, uma vez que a sua justificação é aduzida dos problemas em estudo, e não da descrição de PAF.

\section{Padrão de atividade física em crianças e jovens.}

Dos 26 estudos que atenderam aos critérios de inclusão, 18 foram realizados em populações europeias, cinco na população norte americana, e três em outras populações. Os sumários relativos às características principais das pesquisas estão nas tabelas 1 à 3 .

Quadro 1. Estudos sobre padrão de atividade física (PAF) realizados na população europeia entre os anos de 2000 a 2008.

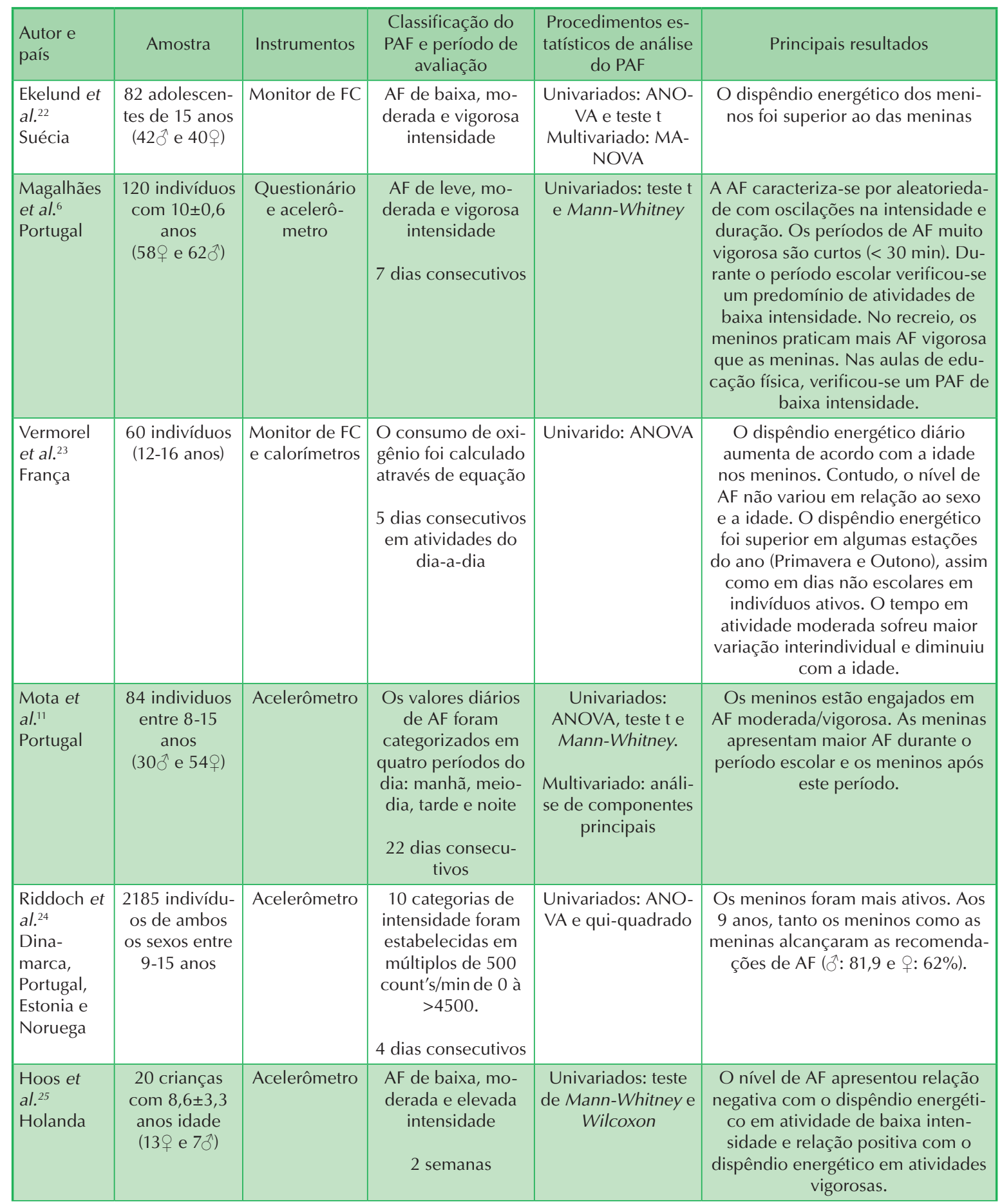




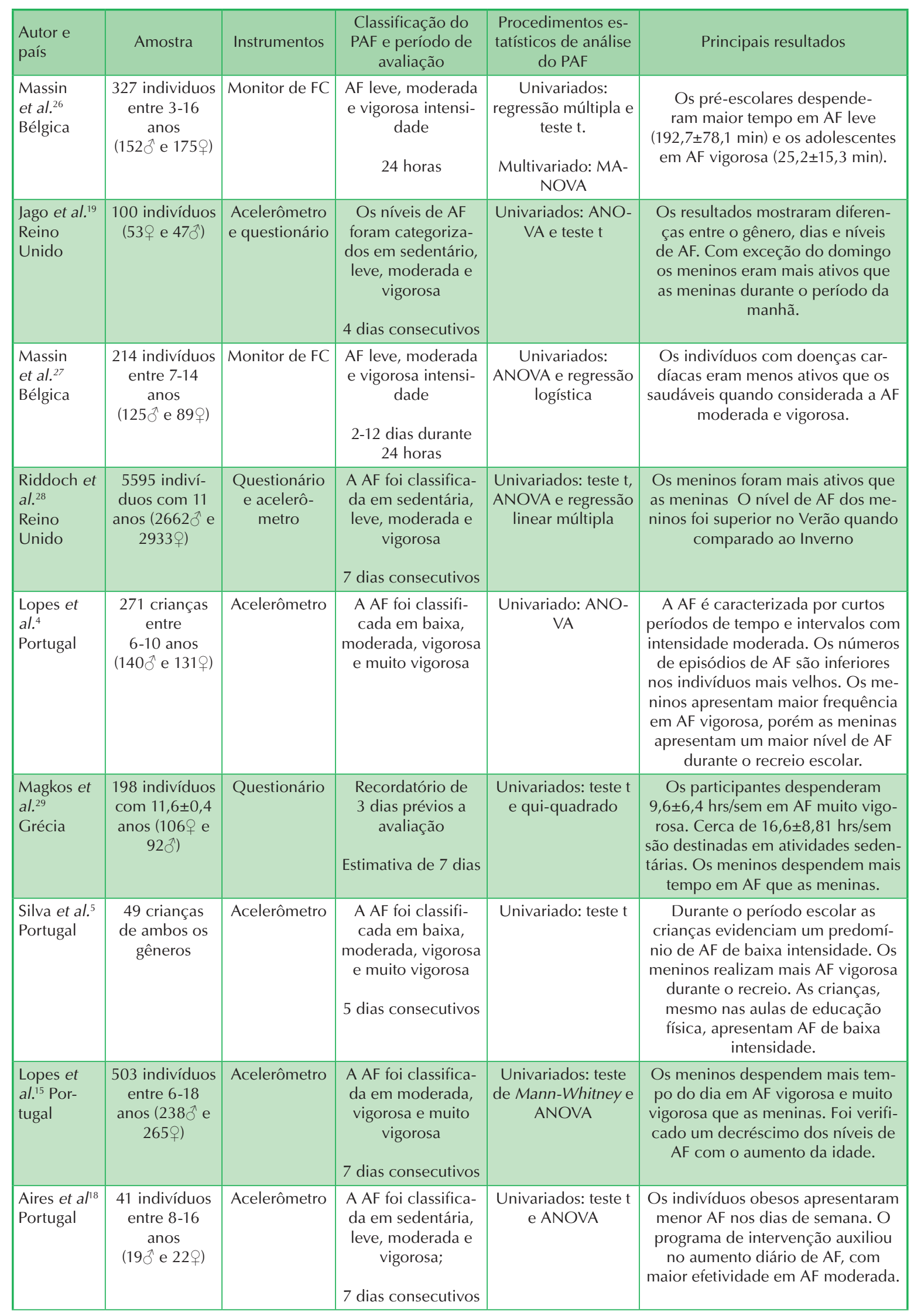




\begin{tabular}{|c|c|c|c|c|c|}
\hline $\begin{array}{l}\text { Autor e } \\
\text { país }\end{array}$ & Amostra & Instrumentos & $\begin{array}{l}\text { Classificação do } \\
\text { PAF e período de } \\
\text { avaliação }\end{array}$ & $\begin{array}{l}\text { Procedimentos es- } \\
\text { tatísticos de análise } \\
\text { do PAF }\end{array}$ & Principais resultados \\
\hline $\begin{array}{l}\text { Sigmund et } \\
\text { al. }{ }^{30} \\
\text { República } \\
\text { Checa }\end{array}$ & $\begin{array}{l}2065 \text { indiví- } \\
\text { duos dos 5-24 } \\
\text { anos }\end{array}$ & Acelerômetro & $\begin{array}{c}\text { O dispêndio } \\
\text { energético foi } \\
\text { calculado levando } \\
\text { em consideração a } \\
\text { frequência, tipo, in- } \\
\text { tensidade e tempo } \\
\text { em AF } \\
7 \text { dias consecutivos }\end{array}$ & $\begin{array}{l}\text { Multivariado: MA- } \\
\text { NOVA }\end{array}$ & $\begin{array}{l}\text { O dispêndio energético dos pré- } \\
\text { escolares foi superior aos dos ado- } \\
\text { lescentes e adultos. O nível de AF } \\
\text { dos pré-escolares não variou entre } \\
\text { os dias, ao contrário dos demais } \\
\text { grupos. }\end{array}$ \\
\hline $\begin{array}{l}\text { Bathrellou } \\
\text { et al. }{ }^{21} \\
\text { Grécia }\end{array}$ & $\begin{array}{c}1140 \text { indivídu- } \\
\text { os entre } 10-12 \\
\text { anos }(531 \delta \mathrm{e} \\
609+)\end{array}$ & Questionário & $\begin{array}{l}\text { A AF foi classifica- } \\
\text { da em função do } \\
\text { tempo }(0-\geq 8 \text { hrs/ } \\
\text { dia) e intensida- } \\
\text { de (moderada e } \\
\quad \text { vigorosa) } \\
1 \text { semana escolar }\end{array}$ & $\begin{array}{l}\text { Univariados: teste t } \\
\text { e Mann-Whitney }\end{array}$ & $\begin{array}{l}\text { As crianças do meio rural repor- } \\
\text { taram praticar AF após o período } \\
\text { escolar e em ocupações semanais } \\
\text { com tarefas ao ar livre. Os indivídu- } \\
\text { os que residiam em centros urbanos } \\
\text { reportaram mais AF durante a se- } \\
\text { mana. Entretanto, o tempo semanal } \\
\text { dispendido em AF não foi diferente. }\end{array}$ \\
\hline $\begin{array}{l}\text { Rowlands } \\
\text { et al. }{ }^{31} \\
\text { Reino } \\
\text { Unido }\end{array}$ & $\begin{array}{l}84 \text { indivíduos } \\
\text { entre } 9-11 \\
\text { anos } \\
(39+\text { e } 45 \delta)\end{array}$ & Acelerômetro & $\begin{array}{l}\text { A AF foi classifica- } \\
\text { da em ligeira, mo- } \\
\text { derada e vigorosa } \\
7 \text { dias consecutivos }\end{array}$ & $\begin{array}{l}\text { Univariados: } \\
\text { ANOVA }\end{array}$ & $\begin{array}{c}\text { A frequência, duração e intensidade } \\
\text { da AF é maior nos meninos }\end{array}$ \\
\hline
\end{tabular}

FC: frequência cardíaca; AF: atividade física; PAF: padrão de atividade física

Quadro 2. Estudos sobre padrão de atividade física (PAF) realizados na população norte-americana entre os anos de 2000 a 2007.

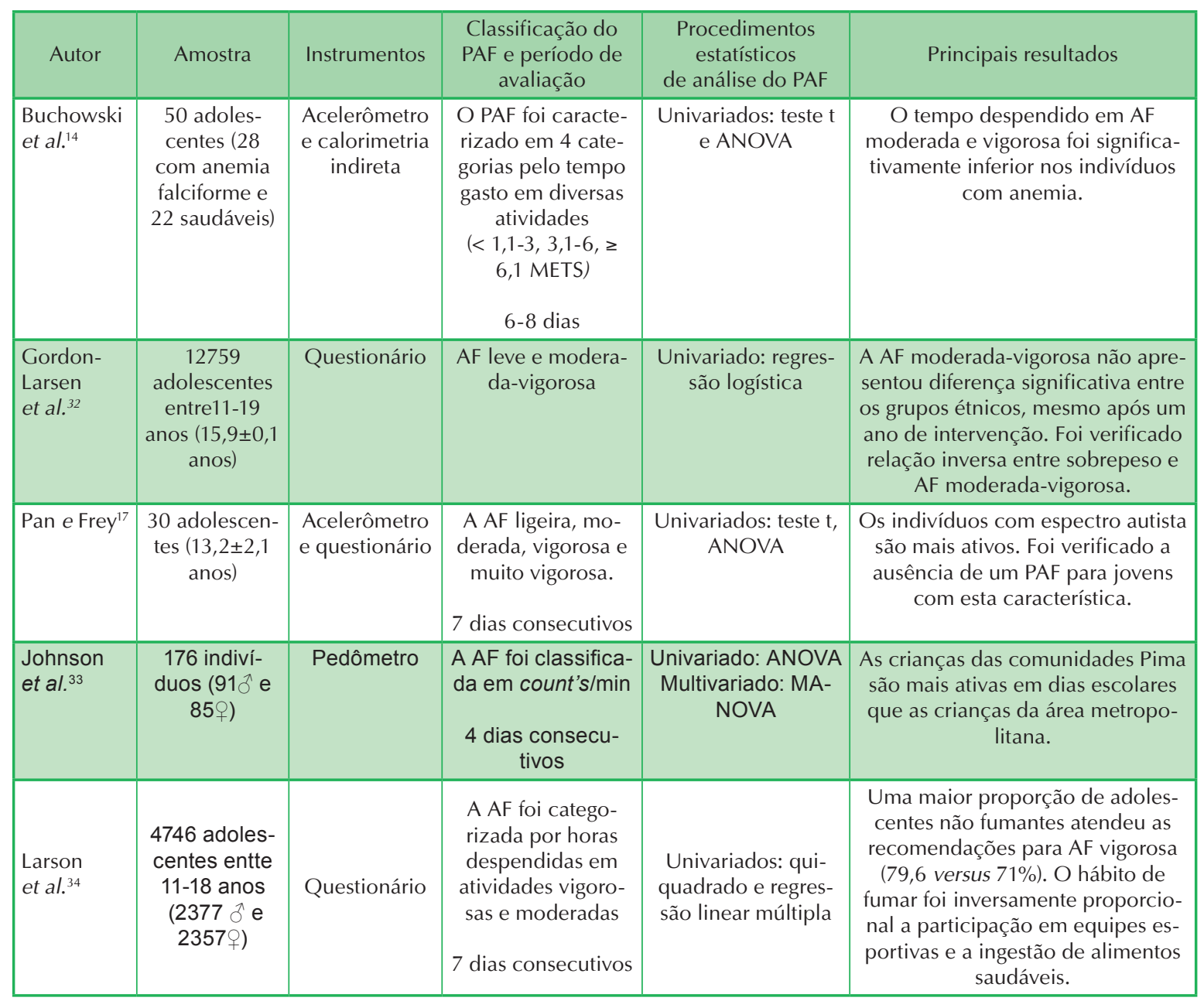

PAF: padrão de atividade física; AF: atividade física. 
Quadro 3. Estudos sobre padrão de atividade física (PAF) realizados em outras populações entre os anos de 2004 a 2007.

\begin{tabular}{|c|c|c|c|c|c|}
\hline Autor e país & Amostra & Instrumentos & $\begin{array}{l}\text { Classificação do PAF e } \\
\text { período de avaliação }\end{array}$ & $\begin{array}{c}\text { Procedimentos } \\
\text { estatísticos } \\
\text { de análise do PAF }\end{array}$ & Principais resultados \\
\hline $\begin{array}{l}\text { Janssen et } \\
\text { al. }^{35} \\
\text { Canadá }\end{array}$ & $\begin{array}{c}5890 \text { adoles- } \\
\text { centes entre } 11- \\
16 \text { anos }(2812 \AA \\
\text { e } 3078+)\end{array}$ & Questionário & 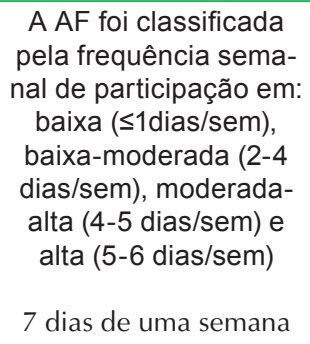 & $\begin{array}{c}\text { Univariados: } \\
\text { qui-quadrado e } \\
\text { regressão logística } \\
\text { múltipla }\end{array}$ & $\begin{array}{l}\text { Indivíduos obesos } \\
\text { apresentaram menor fre- } \\
\text { quência em AF e maior } \\
\text { utilização da televisão } \\
\text { que aqueles com so- } \\
\text { brepeso. Foi verificado } \\
\text { relação negativa entre a } \\
\text { participação em AF e os } \\
\text { valores de IMC. }\end{array}$ \\
\hline $\begin{array}{l}\text { Sabbah et } \\
\text { al. } \\
\text { Palestina }\end{array}$ & $\begin{array}{c}8885 \text { indivídu- } \\
\text { os }\left(4313{ }^{\wedge} \mathrm{e}\right. \\
4572+)\end{array}$ & Questionário & $\begin{array}{c}\text { A AF foi classificada } \\
\text { pela frequência sema- } \\
\text { nal de participação: } \geq 5 \\
\text { dias/sem e }<5 \text { dias/sem }\end{array}$ & $\begin{array}{l}\text { Univariado: qui- } \\
\text { quadrado }\end{array}$ & $\begin{array}{l}\text { Os meninos são mais } \\
\text { ativos que as meninas. } \\
\text { Foi verificado uma } \\
\text { diminuição da AF com o } \\
\text { incremento da idade. }\end{array}$ \\
\hline $\begin{array}{l}\text { Yamauchi et } \\
\text { al. }{ }^{37} \\
\text { Japão }\end{array}$ & $\begin{array}{l}159 \text { indivíduos } \\
\text { entre } 10-14 \\
\text { anos } \\
(20 \hat{\jmath} \text { e } 209)\end{array}$ & Acelerômetro & $\begin{array}{l}\text { Foi calculado o nível } \\
\text { de AF e a sua variação } \\
\text { diária. } \\
7 \text { dias consecutivos }\end{array}$ & $\begin{array}{l}\text { Univariado: teste t } \\
\text { Bivariado: correla- } \\
\text { ção de Pearson }\end{array}$ & $\begin{array}{l}\text { As meninas tendem a } \\
\text { ter maior nível de AF } \\
\text { que os meninos. Os } \\
\text { indivíduos foram menos } \\
\text { ativos aos domingos }\end{array}$ \\
\hline
\end{tabular}

AF: atividade física, IMC: índice de massa corporal

Na população europeia, os resultados apresentados com recurso de acelerômetros mostraram que o nível de AF de meninos e meninas está abaixo do recomendado. Os meninos são mais ativos que as meninas, dos nove aos 15 anos de idade ${ }^{24}$. No tempo despendido em AF moderada, as diferenças são evidentes aos nove e aos 15 anos, sendo que os meninos apresentam maior gasto energético em relação às meninas ${ }^{24,31}$, adolescentes e adultos ${ }^{24}$.

Em estudos com o auxílio de monitores de FC $^{22,23,26,27}$, os valores não foram muito diferentes, salientando um declínio da AF com aumento da idade e os meninos sendo mais ativos. Também foi verificado o fato da $A F$ não se caracterizar por períodos prolongados e sim, por pequenos períodos de atividade moderada e enorme aleatoriedade ${ }^{22}$. Esses resultados também são verificados pelos trabalhos que utilizaram questionários ${ }^{29,36}$. Na população norte americana, os valores não se apresentaram diferentes dos europeus. Larson et al..$^{34}$, em um estudo realizado com adolescentes fumantes, reportaram que a AF é inversamente proporcional ao número de cigarros consumidos por dia e que os não-fumantes praticam mais AF vigorosa. Gordon-Larsen et al. ${ }^{32}$ mostraram que o sobrepeso decresce com o aumento da frequência de AF moderada e vigorosa em meninos e meninas de diferentes origens étnicas. Os estudos encontrados com populações de outros países revelaram os benefícios da AF para o controle do IMC ${ }^{35,37}$. Foi verificado que o nível de AF varia de dia para $\mathrm{dia}^{20}$, porém nenhum destes estudos apresentou evidências bem claras de uma definição concisa e distinta do que os autores entendem por PAF.

Da revisão dos estudos anteriores não emergiu uma definição clara e concisa do que se entende pela expressão "padrão de AF", dado ser comumente utilizada como sinônimo de AF habitual, AF diária ou ainda, níveis de AF. Não obstante a importância dos resultados disponíveis, da carência consensual conceitual e operativa de PAF, deve ter-se em linha de conta algumas insuficiências da presente pesquisa: (1) a circunstância de ter limitado a inventariação de trabalhos a partir do ano 2000; (2) ter considerado somente crianças e adolescentes; (3) de não ser ainda clara a relevância do PAF em termos da sua associação com aspectos dos estilos de vida mais saudáveis, algumas morbilidades e comportamentos de risco; (4) pela dificuldade que é contrastar níveis ou PAF provenientes de instrumentos altamente díspares, e (5) pela diversidade de procedimentos estatísticos utilizados.

\section{CONSIDERAÇÕES FINAIS}

Os resultados da revisão mostraram que a expressão padrão de AF é comumente utilizada, porém não é encontrada uma interpretação e conceitualização unânime, tampouco uma definição operacional única. Acrescentamos a presença de diferentes técnicas de análise estatística que torna difícil a comparação de resultados entre pesquisas. Foi genericamente constatado que o PAF em crianças 
e jovens se caracteriza por períodos extremamente curtos de alta intensidade, com predomínio de atividades de baixa a moderada intensidade. Aos finais de semana, esses períodos são maiores e os meninos apresentam uma maior frequência de episódios de níveis distintos de intensidade.

É urgente a obtenção de um conjunto informacional mais extenso acerca da noção de PAF. $\mathrm{O}$ recurso à avaliação multimodal (considerando, em simultâneo, dados de acelerômetros, diários, questionários e pedômetros) poderia ser de grande utilidade, sobretudo, na interpretação e alcance dos tipos, níveis, frequências e durações da AF e respectivas variações bem descritas pelo PAF. $\mathrm{O}$ maior desafio vai para a descrição e atribuição de significado aos padrões emergentes (obtidos de séries temporais) e a sua associação às crianças obesas e outras com distintas co-morbilidades. Decorreria daqui uma interpretação bem esclarecedora das suas ligações a outros preditores importantes de natureza genética e ambiental, cujas implicações reclamariam programas de intervenção mais adequados em termos específicos e de rotinas de vida mais ativa e saudável de crianças e adolescentes.

\section{REFERÊNCIAS BIBLIOGRÁFICAS}

1. Strong WB, Malina RM, Blimkie CJ, Daniels SR, Dishman RK, Gutin B, et al. Evidence based physical activity for school-age youth. J Pediatr 2005;146(6):732-737.

2. Sallis JF, Patrick K. Physical activity guidelines for adolescents: consensus statement. Pediatr Exerc Sci 1994;6(4):302-314.

3. Twisk JW. Physical activity guidelines for children and adolescents: a critical review. Sports Med 2001;31(8):617-627.

4. Lopes VP, Vasques CMS. Physical activity patterns during school recess: A study in children 6 to 10 years old. Int Elect J Health Educ 2006;9:192-201.

5. Silva SP, Magalhães M, Garganta RM, Seabra A, Bustamente A, Maia JAR. Padrão de actividade física de escolares. Rev Bras Cineantropom Desempenho Hum 2006;8(2):19-26.

6. Magalhães L, Maia JAR, Silva R, A. S. Padrão de actividade física. Estudo em crianças de ambos os sexos do $4^{\circ}$ ano de escolaridade. Rev Port Cien Desp 2002;2(5):47-57.

7. The New Oxford Dictionary of English. Oxford University Press; 1998.

8. Houaiss A, Villar MS. Dicionário Houaiss da língua portuguesa: Círculo de leitores. Editora: Temas e Debates; 2003.

9. Van Der Horst K, Paw MJ, Twisk JW, Van Mechelen W. A brief review on correlates of physical activity and sedentariness in youth. Med Sci Sports Exerc 2007;39(8):1241-1250.
10. Sallo M, Silla R. Physical activity with moderate to vigorous intensity in preschool and first-grade schoolchildren. Pediatr Exerc Sci 1997;9(1):44-54.

11. Mota J, Santos P, Guerra S, Ribeiro JC, Duarte JA. Patterns of daily physical activity during school days in children and adolescents. Am J Biol 2003;15(4):547-553.

12. Lopes VP, Maia JAR, Oliveira MMC, Seabra A, Garganta R. Caracterização da atividade física habitual em adolescentes de ambos os sexos através da acelerometria e pedometria. Rev Paul de Educ Física 2003;17(1):51-63.

13. Lopes VP, Maia JAR. Actividade física nas crianças e jovens. Rev Bras Cineantropom Desempenho Hum 2004;6(1):82-92.

14. Buchowski MS, Townsend KM, Williams R, Chen KY. Patterns and energy expenditure of free-living physical activity in adolescents with sickle cell anemia. J Pediatr 2002;140(1):86-92.

15. Lopes VP, Vasques CMS, Maia JAR, Ferreira JCV. Habitual physical activity levels in childhood and adolescents assessed with accelerometry. J Sports Med Phys Fit 2007;47(2):217-222.

16. Fox KR, Riddoch C. Charting the physical activity patterns of contemporary children and adolescents. Proc Nutr Soc 2000;59:497-504.

17. Pan CY, Frey GC. Physical Activity Pattern in Youth with Autism Spectrum Disorders. J Aut Devel Dis 2006;36(5):597-606.

18. Aires L, Santos R, Silva P, Santos P, Oliveira J, Ribeiro JC, et al. Daily differences in patterns of physical activity among overweight/obese children engaged in a physical activity program. Am J Hum Biol 2007;19(6):871-877.

19. Jago R, Anderson CB, Baranowski T, Watson K. Adolescents patterns of physical activity. Differences by gender, day, and time of day. Am J Prev Med 2005;28(5):447-452.

20. Ndiaye GM, Bénéfice E. Patterns of daily activity and time spent in bed of adult woman and adolescents and preadolescents girls from a rural community in Senegal, West Africa. Ann Hum Biol 2007;34(4):454-469.

21. Bathrellou E, Lazarou C, Panagiotakos DB, Sidossis LS. Physical activity patterns and sedentary behaviors of children from urban and rural areas of Cyprus. Eur J Public Health 2007;15(2):66-70.

22. Ekelund U, Sjöström M, Yngve A, Nilsson A. Total daily energy expenditure and pattern of physical activity measured by minute-by-minute heart rate monitoring in 14-15 year old Swedish adolescents. Eur J Clin Nutr 2000;54(3):195-202.

23. Vermorel M, Vernet J, Bitar A, Fellmann N, Coudert J. Daily energy expenditure, activity patterns, and energy costs of the various activities in French 12-16-y-old adolescents in free living conditions. Eur J Clin Nutr 2002;56(9):819-829.

24. Riddoch CJ, Andersen LB, Wedderkopp N, Harro M, Klasson-Heggebo L, Sardinha LB. Physical activity levels and patterns of 9 and 15-yr-old european children. Med Sci Sports Exerc 2004;36(1):86-92. 
25. Hoos MB, Kuipers H, Gerver W-J, Westerterp KR. Physical activity patterns of children assessed by triaxial accelerometry. Eur J Clin Nutr 2004;58(10):1425-1428.

26. Massin MM, Lebrethon MC, Rocour D, Gérard P, Bourguignon JP. Patterns of physical activity determined by heart rate monitoring among diabetic children. Arch Dis Children 2005;90(12):1223-1226.

27. Massin MM, Hövels-Gürich HH, Gérard P, Seghaye MC. Physical activity patterns of children after neonatal arterial switch operation. Ann Thorac Surg 2006;81(2):665-670.

28. Riddoch CJ, Mattocks C, Deere K, Saunders J, Kirkby J. Objective measurement of levels and patterns of physical activity. Arch Dis Childhood 2007;92(11):963-969.

29. Magkos F, Piperkou I, Manios Y, Papoutsakis C, Yiannakouris N, Cimponeiro A, et al. Diet, blood lipid profile and physical activity patterns in primary school children from a semi-rural area of Greece. J Hum Nutr Diet 2006;19(2):101-112.

30. Sigmund E, Croix MDS, Miklánková L, Frömel K. Physical activity patterns of kindergarten children in comparison to teenagers and young adults. Eur J Public Health 2007;12(6):646-651.

31. Rowlands AV, Pilgrim EL, Eston RG. Patterns of habitual activity across weekdays and weekend days in 9-11-year-old children. Prev Med 2008;46(4):317-324.

32. Gordon-Larsen P, Adair LS, Popkin BM. Ethnic Differences in Physical Activity and Inactivity Patterns and Overweight Status. Obes Res 2002;10(3):141-149.

33. Johnson TG, Kulinna PH, Darst PW, Pangrazi RP. School day physical activity patterns of Pima indian children in two communities. Res Quart Exerc Sport 2007;78(4):364-368.
34. Larson NI, Story M, Perry CL, Neumark-Sztainer D, Hannan PJ. Are diet and physical activity patterns related to cigarette smoking in adolescents? findings from project EAT. Prev Chro Dis Public Health Res 2007;4(3):A51.

35. Janssen I, Katzmarzyk PT, Boyce WF, King MA, Pickett W. Overweight and obesity in canadian adolescents and their association with dietary habits and physical activity patterns. J Adol Health 2004;35(5):360-367.

36. Sabbah HAL, Vereecken C, Kolsteren P, Abdeen Z, Maes L. Food habits and physical activity patterns among Palestinian adolescents: findings from the national study of Palestinian schoolchildren (HBSCWBG2004). Public Health Nutr 2007;10(7):739-746.

37. Yamauchi T, Kim S-N, Lu Z, Ichimaru N, Maekawa R, Natsuhara K. Age and gender differences in the physical activity patterns of urban schoolchildren in Korea and China. J Physi Anthr 2007;26(2):101-107.

\author{
Endereço para correspondência \\ José António Ribeiro Maia \\ Laboratório de Cineantropometria e Gabinete \\ de Estatística Aplicada \\ Universidade do Porto - Faculdade de Desporto \\ Rua Plácido Costa, 91 - 4200-450 \\ Porto, Portugal \\ E-mail: jmaia@fade.up.pt
}

